\title{
Correction to: Exogenous toluene gas removal improvement in recombinant rabbit cytochrome P450 2E1 (CYP2E1)-transgenic Ardisia pusilla DC
}

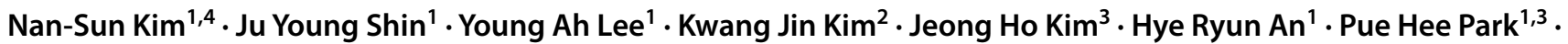 \\ Pil Man Park ${ }^{1} \cdot$ Su Young Lee ${ }^{1}$
}

Published online: 14 April 2021

(c) Korean Society for Horticultural Science 2021

\section{Correction to: \\ Horticulture, Environment, and Biotechnology \\ https://doi.org/10.1007/s13580-021-00333-2}

The original version of this article unfortunately contained a mistake. The following correction has therefore been made in the original: The spelling of Su Young Lee's name was incorrect. The corrected author list is given above. The original article has been corrected.

Publisher's Note Springer Nature remains neutral with regard to jurisdictional claims in published maps and institutional affiliations.

The original article can be found online at https://doi.org/10.1007/ s13580-021-00333-2.

Su Young Lee

1sy8542224@korea.kr

1 Floriculture Research Division, National Institute of Horticultural and Herbal Science, Rural Development Administration, Wanju 55365, South Korea

2 Urban Agriculture Research Division, National Institute of Horticultural and Herbal Science, Rural Development Administration, Wanju 55365, South Korea

3 Planning and Coordination Division, National Institute of Horticultural and Herbal Science, Rural Development Administration, Wanju 55365, South Korea

4 Metabolic Engineering Division, National Institute of Agricultural Sciences, Rural Development Administration, Wanju 55365, South Korea 